\title{
Salicylate-Induced Changes in Hearing Thresholds in Mongolian Gerbils Are Correlated With Tinnitus Frequency but Not With Tinnitus Strength
}

\section{OPEN ACCESS}

Edited by: Joseph P. Walton, University of South Florida,

United States

Reviewed by:

Alexander Galazyuk, Northeast Ohio Medical University,

United States

Veronika Vielsmeier, ENT-Clinic University of Regensburg, Germany

*Correspondence:

Holger Schulze

holger.schulze@uk-erlangen.de

Specialty section:

This article was submitted to

Pathological Conditions,

a section of the journal

Frontiers in Behavioral Neuroscience

Received: 21 April 2021

Accepted: 07 July 2021

Published: 30 July 2021

Citation:

Lanaia V, Tziridis $K$ and Schulze H (2021) Salicylate-Induced Changes in Hearing Thresholds in Mongolian Gerbils Are Correlated With Tinnitus

Frequency but Not With Tinnitus

Strength.

Front. Behav. Neurosci. 15:698516. doi: 10.3389/fnbeh.2021.698516

\section{Veralice Lanaia, Konstantin Tziridis and Holger Schulze*}

Experimental Otolaryngology, University Hospital Erlangen, Friedrich-Alexander University Erlangen-Nürnberg, Erlangen, Germany

Tinnitus is an auditory phantom percept without external sound sources. Despite the high prevalence and tinnitus-associated distress of affected patients, the pathophysiology of tinnitus remains largely unknown, making prevention and treatments difficult to develop. In order to elucidate the pathophysiology of tinnitus, animal models are used where tinnitus is induced either permanently by noise trauma or transiently by the application of salicylate. In a model of trauma-induced tinnitus, we have suggested a central origin of tinnitus-related development of neuronal hyperactivity based on stochastic resonance (SR). SR refers to the physiological phenomenon that weak subthreshold signals for given sensors (or synapses) can still be detected and transmitted if appropriate noise is added to the input of the sensor. The main objective of this study was to characterize the neurophysiological and behavioral effects during salicylate-induced tinnitus and compare these to the conditions within the trauma model. Our data show, in line with the pharmacokinetics, that hearing thresholds generally increase $2 \mathrm{~h}$ after salicylate injections. This increase was significantly stronger within the region of best hearing compared to other frequencies. Furthermore, animals showed behavioral signs of tinnitus during that time window and frequency range as assessed by gap prepulse inhibition of the acoustic startle reflex (GPIAS). In contrast to animals with noise trauma-induced tinnitus, salicylate-induced tinnitus animals showed no correlation between hearing thresholds and behavioral signs of tinnitus, indicating that the development of tinnitus after salicylate injection is not based on SR as proposed for the trauma model. In other words, salicylate-induced tinnitus and noise trauma-induced tinnitus are not based on the same neurophysiological mechanism.

Keywords: tinnitus, effect size, salicylate, noise trauma, stochastic resonance, hearing threshold

\section{INTRODUCTION}

Diseases of the inner ear that lead to hearing loss (HL) may also result in subjective tinnitus (Ahlf et al., 2012), an auditory phantom sensation that is experienced, although no physical sound is present. Tinnitus occurs with surprisingly high prevalence, affecting about $35 \%$ (Shargorodsky et al., 2010) of the general population, with $10-15 \%$ of individuals experiencing 
prolonged periods of tinnitus that require medical evaluation. For $10 \%$ of the population, tinnitus has a significant impact on their quality of life (Chao et al., 2014). Despite this high prevalence and the tinnitus-associated distress of affected patients, which, in severe cases, may experience insomnia, psychological disorders like depression, the inability to work, or even commit suicide (Coles, 1984; Lewis et al., 1994; Langguth et al., 2011), the cause(s) and pathophysiology of tinnitus are still controversially discussed, making prevention and treatments difficult to develop (Turner et al., 2006). More than 20 years ago, tinnitus was thought to result from aberrant neural activity generated in the periphery of the auditory system (Jastreboff, 1990). In particular, tinnitus was proposed to result from increased activity in the cochlear nerve. More recently, due to the developments in basic neuroscience, a central origin of tinnitus-related activity seems to have replaced the former peripheral hypothesis (Noreña and Farley, 2013). The main reason for this shift is based on evidence that surgical neurotomy of the cochlear nerve, which should suppress tinnitus if the activity driving the percept originated in the cochlear nerve, has hardly ever had this intended effect (House and Brackman, 1981; Barrs and Brackmann, 1984; Silverstein et al., 1986; Pulec, 1995; Baguley et al., 2002). An effective cure for tinnitus still does not exist, and the main reason is that the neurophysiological mechanism that leads to the development of tinnitus is still not fully understood. Until today, several tinnitus models are being debated, although, due to developments in basic neuroscience, a central origin of tinnitusrelated activity seems to have replaced the former peripheral hypothesis (Noreña and Farley, 2013). In particular, three main models, which are based on altered lateral inhibition, homeostatic plasticity, or stochastic resonance (SR) (Gerken, 1996; Eggermont and Roberts, 2004; Schaette and McAlpine, 2011; Ahlf et al., 2012; Tziridis et al., 2015; Leaver et al., 2016; Vanneste and De Ridder, 2016; Schilling et al., 2020), propose a central origin of tinnitus, resulting from damage to the cochlea as the initial step in tinnitus development.

In our recent work, Krauss and colleagues (Krauss et al., 2016) have suggested a central origin of tinnitus-related development of neuronal hyperactivity based on SR, which refers to the phenomenon that weak signals, which are subthreshold for a given sensor (or synapse), can still be detected and transmitted by that sensor if (neuronal) noise is added to the sensor input. We assumed that SR at the level of the dorsal cochlear nucleus constantly optimizes information transmission into the auditory system and, thereby, may, e.g., compensate for hearing loss. In this view, the noise necessary for SR is then the neurophysiological source of tinnitus-related enhanced neuronal activity.

The two main tinnitus inducers in humans are noise trauma (Chermak and Dengerink, 1987; Metternich and Brusis, 1999; Temmel et al., 1999; Stankiewicz et al., 2000; Mrena et al., 2002; Langguth et al., 2011) and high dose of salicylate (Myers and Bernstein, 1965; McFadden et al., 1984; Day et al., 1989; Cazals, 2000; Baguley et al., 2002; Langguth et al., 2011). In this study, the effect of salicylate is tested in Mongolian gerbils, and the results are compared to data of noise trauma-induced tinnitus (Ahlf et al., 2012; Tziridis et al., 2015) to investigate if those two types of tinnitus are based on the same neurophysiological mechanism or not.

We induce tinnitus in Mongolian gerbils, because, in contrast to mice and rats, the hearing of the gerbil up to $20 \mathrm{kHz}$ closely resembles the human audiogram (best hearing around $4 \mathrm{kHz}$ ) (Ryan, 1976). We, here, compare neurophysiological and behavioral markers of tinnitus in animals receiving a noise trauma at $2 \mathrm{kHz}$ and $115 \mathrm{~dB}$ SPL for $75 \mathrm{~min}$ and animals receiving subcutaneous injections of a high dose of salicylate $(300 \mathrm{mg} / \mathrm{kg})$, as it has been demonstrated before that salicylate doses between 150 and $350 \mathrm{mg} / \mathrm{kg}$ induce tinnitus in rodents (Sheppard et al., 2014). Behavioral estimates of salicylate-and noise traumainduced tinnitus were obtained, using gap prepulse inhibition of the acoustic startle reflex (GPIAS, cf. Schilling et al., 2017), and auditory brainstem responses (ABR) were recorded to monitor changes in central auditory activity. We tested frequency-specific differences in GPIAS after noise trauma and salicylate treatment, and we correlated these data with possible ABR threshold changes in the same animals to evaluate similarities and differences of both tinnitus models. Taken together, the aim of the study was the characterization of neurophysiological and behavioral effects of salicylate-induced tinnitus and its comparison with data obtained with the noise trauma-induced tinnitus in the context of our model of SR-based tinnitus development.

\section{MATERIALS AND METHODS}

\section{Ethics Statement and Animals}

Mongolian gerbils (Meriones unguiculatus) were housed in standard animal racks (Bio A. S. Vent Light, EHRET Laborund Pharmatechnik, Emmendingen, Germany) in groups of 2-3 animals per cage with free access to water and food at $20-24^{\circ} \mathrm{C}$ room temperature under a $12 / 12$-h dark/light cycle. The use and care of animals were approved by the state of Bavaria (reference No. 55.2-2532-2-726, Regierungspräsidium Unterfranken, Würzburg, Germany).

\section{Salicylate Treatment}

A total number of 37 10-week-old male gerbils purchased from Janvier (Saint Berthevin Cedex, France) were used in this study. Eighteen animals were treated with subcutaneous injection of isotonic saline (control group $\mathrm{C}, \sim 0.5 \mathrm{ml}$ ) and 19 animals with subcutaneous injection of sodium salicylate (group S, $300 \mathrm{mg} / \mathrm{kg}$; Sigma), dissolved in the saline, resulting in the same amount of injection volume $(\sim 0.5 \mathrm{ml})$. All animals were examined, using the GPIAS and ABR measurements (Figure 1). We first measured the baseline behavior in the prepulse inhibition (PPI) of the acoustic startle reflex (ASR) of each animal (cf. below). The next day, we measured the audiograms, using pure tone ABR (pure tone hearing threshold, $\mathrm{HT}$ ), first before the injection and, subsequently, $20 \mathrm{~min}$ and $2 \mathrm{~h}$ after the injection in both groups (cf. below). After 7 days, we, again, measured the audiograms of the animals in order to evaluate possible long-term effects of the salicylate treatment on HT. Once it was certain that the effect had disappeared, we proceeded with a second injection of either salicylate or 


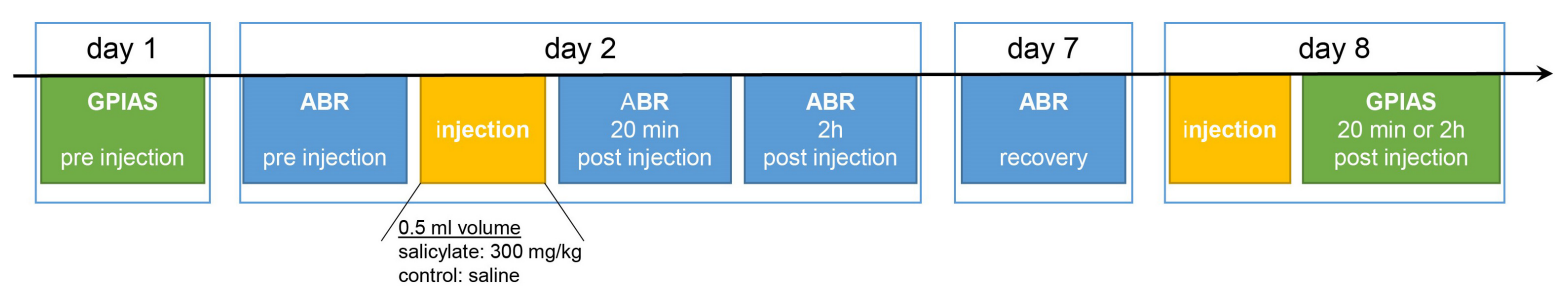

FIGURE 1 | Timeline of experiments. We first measured the baseline behavior in the prepulse inhibition (PPI) of the acoustic startle reflex (ASR) of each animal. The next day, we measured the audiograms, using pure tone ABR (pure tone hearing threshold, $\mathrm{HT}$ ), first before the injection and, subsequently, 20 min and $2 \mathrm{~h}$ after the injection in both groups. After 7 days, we, again, measured the audiograms of the same animals. On the 8th day, we proceeded with a second injection of either salicylate or saline in the same (now awake) animals and obtained the 20-min or 2-h behavior response in the PPI of the ASR.

saline in the same (now awake) animals and obtained the GPIAS again to assess a possible acute tinnitus percept. Control and salicylate animals were separated into two groups based on the temporal delay of the behavioral test after the injection. Nine animals of the control group were behaviorally tested $20 \mathrm{~min}$ after the injection, and another nine control animals were behaviorally tested $2 \mathrm{~h}$ post-injection. In the salicylate group, 10 animals were tested $20 \mathrm{~min}$, following the injection and, again, $2 \mathrm{~h}$, following the injection, and nine animals were behaviorally tested $2 \mathrm{~h}$ post-injection only. There was no significant difference ( $t$-tests with $p$-value between 0.14 and 0.7 ); the 2 - $h$ responses of these animals and were, therefore, treated as one 2-h group. We later analyzed frequency-specific differences in the startle reflex responses after injection and correlated these data with post vs. pre-injection differences in the ABR thresholds (cf. below).

\section{Data of Animals Treated With Acoustic Trauma}

For the comparison of the salicylate with trauma data, we reanalyzed GPIAS and ABR threshold data of 16 animals treated with a binaural acoustic noise trauma of $2 \mathrm{kHz}$ and $115 \mathrm{~dB}$ SPL over 75 min under anesthesia. All methods of data recording are already published (Ahlf et al., 2012; Tziridis et al., 2015). In a nutshell, the trauma for tinnitus induction was applied under deep ketamine-xylazine anesthesia as described in detail earlier (Ahlf et al., 2012; Walter et al., 2012; Tziridis et al., $2014,2015)$. The anesthetized animals were placed on a heating pad with a remote-controlled temperature of $37^{\circ} \mathrm{C}$, centered in front of a loudspeaker (Canton Plus X Series 2; Canton, Weilrod, Germany). Using a signal generator (hp 33120A, HP, Böblingen, Germany) connected to an audio amplifier (Amp 75, ThomasWulf, Frankfurt, Germany), a $2 \mathrm{kHz}$ pure tone was presented at a sound pressure level of $115 \mathrm{~dB}$ SPL for $75 \mathrm{~min}$.

Pre GPIAS and ABR recordings were performed during the week prior to the trauma (cf. Figure 1). The post-trauma ABR was recorded during a 2-h period after the treatment when trauma effects were strongest. The behavioral responses were recorded 5 to 7 days after the trauma when the animals completely recovered from the procedure and a possible tinnitus percept reached its subacute phase. Datasets were analyzed with our improved methods of GPIAS (Schilling et al., 2017) and ABR threshold evaluation (Schilling et al., 2019) (cf. below). Data of both measurements were correlated with each other in the same way as in the salicylate/saline animals.

\section{Behavioral and ABR Measurements}

All methods used in this paper have been described previously (Tziridis et al., 2015; Schilling et al., 2017, 2019) but will be explained shortly here for better intelligibility.

\section{Auditory Brainstem Response}

As described by Schilling and coworkers (Schilling et al., 2019), ABR measurements were recorded, using a custom-made setup. Pure tone stimuli of different frequencies, ranging from 1 to $8 \mathrm{kHz}$, were generated by a custom-made Python program (Python 3.6.0 and presented at different intensities, ranging from 30 to $90 \mathrm{~dB}$ SPL in $5 \mathrm{~dB}$ steps. Stimulation was performed free-field via a speaker (Sinus Live NEO), corrected for its frequency transfer function to be flat within $\pm 1 \mathrm{~dB}$ at a distance of $\sim 3 \mathrm{~cm}$ from the pinna of the animal. To compensate for speaker artifacts, stimuli were presented in double trials, consisting of two 6-ms stimuli (including 2-ms sine square rise and fall ramps) of the same amplitude but an opposite phase, separated by $100 \mathrm{~ms}$ of silence. A number of 250 trials of each combination of intensity and frequency were presented pseudorandomly at an interstimulus interval of $500 \mathrm{~ms}$. Mongolian gerbils were anesthetized with a mixture of ketamine (Ketaset $100 \mathrm{mg} / \mathrm{ml}$ ) and medetomidine (Dorbene $1 \mathrm{mg} / \mathrm{ml}$ ) (mixture of ketamine $75 \mathrm{mg} / \mathrm{kg} \mathrm{BW}$; medetomidine $0.5 \mathrm{mg} / \mathrm{kg}$ $\mathrm{BW}$; atropine sulfate $0.3 \mathrm{mg} / \mathrm{kg} \mathrm{BW}$ in saline. Deep anesthesia was ensured by an initial subcutaneous injection of $0.4 \mathrm{ml}$ of the anesthetic solution and maintained by application of $0.1 \mathrm{ml} / \mathrm{h}$. During measurements, the animals were placed on a feedbackcontrolled heating pad at $37^{\circ} \mathrm{C}$ to maintain body temperature. Data were recorded, using three silver electrodes positioned subcutaneously, one for grounding at the back of the animals, one reference electrode at the forehead, and the measuring electrode infra-auricular, overlying the bulla of the recording side of the left ear. The potential difference between reference and measuring electrode was amplified by a low-noise amplifier (JHM NeuroAmp 401, J. Helbig Messtechnik, Mainaschaff, Germany; amplification 10,000; bandpass filter 400 to 2,000 Hz and $50 \mathrm{~Hz}$ notch filter). The output signal of the amplifier was digitalized and recorded by an analog-digital converter card (National Instruments Corporation, Austin, TX, United States) with a 
sampling rate of $20 \mathrm{kHz}$ and synchronized with the stimulation via the trigger signal from the stimulation computer. Raw data of 250 double trials per sound level for each stimulus frequency were averaged. Finally, these averaged responses of the two singlephase-inverted stimuli within one double trial were averaged to eliminate stimulus artifacts. From this average, artifact-corrected data, the root mean square (RMS) amplitude values from 0 to $10 \mathrm{~ms}$ after the stimulus onset were calculated to obtain a measure of response strength for each stimulus presented. The HT of the animals was automatically estimated before and after the injection of salicylate or saline (Schilling et al., 2019). Furthermore, the hearing loss (HL), i.e., the difference between the HT values after the injection minus the values of the threshold before the injection was calculated.

\section{Behavioral Assessment of Tinnitus}

As described by Schilling and coworkers (Schilling et al., 2017), the animals were placed in a transparent acrylic tube (length, $10 \mathrm{~cm}$; inner diameter, $4.3 \mathrm{~cm}$ ), which was positioned at distance of $10 \mathrm{~cm}$ in front of a loudspeaker (Canton Plus X Series 2), on a low-vibration table (TMC, Peabody, MA, United States). The whole setup was placed in an acoustic chamber (Industrial Acoustics Company GmbH, Niederkrüchten, Germany). The startle response was measured by a sensor platform with three integrated acceleration sensors (ADXL 335 on GY 61 board, Robotpark). All calibration measurements were made in the restrainer to correct for acoustical perturbations. The front end of the tube was closed with a stainless steel grate (wire mesh, width $0.5 \mathrm{~mm}$ ), allowing for acoustic stimulation with no detectable distortion within the used frequency range of stimulation (a signal-to-noise ratio of at least $70 \mathrm{~dB}$ ). Sound pressure level (SPL) was calibrated, using a condenser microphone (Brüel and Kjaer Type 4190) via a preamplifier (Brüel and Kjaer Type 2669) and a measuring amplifier (Brüel and Kjaer Type 2610). Stimulus generation and data acquisition used custom-made programs (Python, Version 3.6.0) (Gerum et al., 2019). As startle amplitudes tend to be higher for the first few trials, five startle stimuli were presented before the beginning of each measurement to rule out strong habituation effects. For sound generation, the frequency response function of the speaker was calibrated to produce an output spectrum that was flat within $\pm 1 \mathrm{~dB}$. The animals were placed in the tube, in which they fit well and were able to move back and forth roughly $2 \mathrm{~cm}$. We had allowed 10-min habituation time before the GPIAS paradigm started (Turner et al., 2006).

Gap prepulse inhibition of the acoustic startle reflex was used to assess the possible existence of a tinnitus percept and to give a rough estimate of the perceived tinnitus frequencies. The animals were subjected to continuous band pass-filtered $60 \mathrm{~dB}$ SPL loud background noise (2-ms cosine square rise and fall times) with medium frequencies of $1,2,4$, or $8 \mathrm{kHz}$ and a bandwidth of \pm half an octave. The mean duration of the background noise before the startle noise burst was $10 \pm 2.5 \mathrm{~s}$; it ended at the beginning of the startle noise burst. The startle white noise burst (115 dB SPL, $20 \mathrm{~ms}$ ) was presented either $50 \mathrm{~ms}$ after a 50-ms-long silent gap (2-ms cosine square rise and fall times) in the background noise, or it was presented without any gap. The twitching response of animals to the startle stimulus was recorded as described above. For each background frequency, 40 repetitions with and without gap were presented in randomized order. A single session of the GPIAS experiment took roughly $50 \mathrm{~min}$. Every animal was at least tested two times, the first time before any treatment and the second time either $20 \mathrm{~min}$ and/or $2 \mathrm{~h}$ after treatment (cf. above).

The analysis of the behavioral data was performed as described by Schilling and coworkers (Schilling et al., 2017). As the response amplitudes of the PPI of the ASR are not normally distributed, the data were first log-normalized. Then, we exploited the full combinatorial power of all normalized response amplitudes to obtain the PPI distributions before and after manipulation of the animals and calculated the effect size of the behavioral response. Positive values indicate a stronger effect of the gap in the post compared with the precondition. Negative values indicate less effect of the gap after treatment, i.e., a stronger startle response despite the present gap, which is considered to indicate a "filling" of the gap by a tinnitus percept in that frequency range. Additionally, these now normally distributed data could be analyzed, using parametrical statistics, like Student's T-test for comparisons of mean changes, and, therefore, statistically significant changes of the effect size of the PPI change can be used to define the strength of a possible tinnitus percept represented by negative effect size values.

\section{Statistical Analysis}

For statistical analysis, we used Statistica 8 (StatSoft Hamburg, Germany). We performed one-factorial repeated measurement mixed ANOVAs for the variables HT, HL, and effect size over the presented frequencies with the repetition factor time relative to injection for salicylate and the control group separately. Tukey's post hoc test was used to further assess the differences in the data. For the comparison between the control and salicylate groups, we used two-factorial ANOVAs with the factors group and frequency at the three different time points independently. Again, Tukey's post hoc tests were used to further asses the differences in the data. We also investigated the correlations between the effect size and HL by multiple linear regression analyses to assess the underlying neurophysiological dependencies of electrophysiology and behavior.

\section{RESULTS}

\section{Effects of Salicylate Injection on ABR Thresholds}

\section{The Hearing Threshold in the Control Group}

First, the audiograms of 18 control animals (group C) were measured. In detail, the results of a one-factorial repeated measurement mixed ANOVA with the factor frequency and the repetition factor time and interaction of time $X$ frequency are given in Figure 2A. The Figure 2A left panel shows the mean audiogram (factor frequency) averaged across the different time points in control gerbils, with the best hearing frequency at $4 \mathrm{kHz}$. Over time (Figure 2A center panel), we observed a significant decrease of the frequency-averaged hearing threshold (mean pre \pm standard deviation: $38.45 \pm 13.45 \mathrm{~dB}$ SPL; mean 

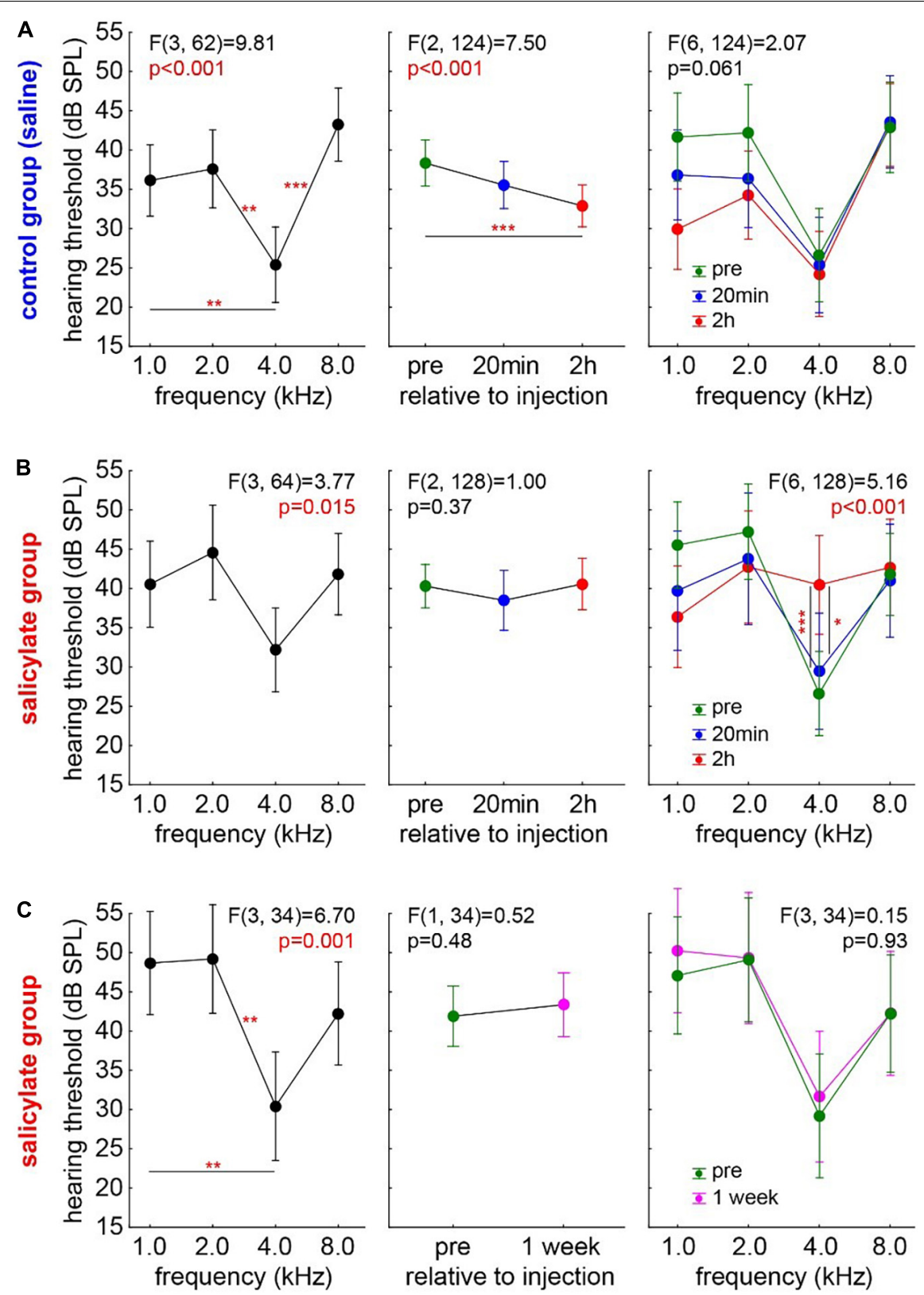

FIGURE 2 | Development of HT over time in both experimental animal groups. One-factorial repeated measurement mixed ANOVA with the factor frequency (left panels) and the repetition factor time (center panels) and interaction of time $X$ frequency (right panels) are shown: (A) Audiogram in the control group (group $\mathrm{C}$, $n=18$ ). Over time, a significant decrease of the frequency-averaged hearing threshold can be observed. No interaction between frequency and time was found. Asterisks indicate a level of Tukey post hoc tests: ${ }^{\star} p<0.05,{ }^{* \star} p<0.01$, ${ }^{\star \star \star} p<0.001$. (B) Audiogram in the salicylate group (group S, $n=19$ ). No HT difference over time was found, but a significant interaction of time $X$ frequency was observed with the strongest effect centered around $4 \mathrm{kHz}$. (C) The transitory effect of the salicylate. No HT differences in the same salicylate animals 1 week post-injection compared with their pre injection recordings.

20 min post saline injection: $35.68 \pm 13.56 \mathrm{~dB}$ SPL; mean, $2 \mathrm{~h}$ post saline injection: $32.94 \pm 12.72 \mathrm{~dB}$ SPL). Post hoc Tukey tests showed $p<0.001$ between pre and $2 \mathrm{~h}$ post saline injection HT, while pre vs. 20 min post-injection and $2 \mathrm{~h}$ vs. $20 \mathrm{~min}$ post-injection HT were not significantly different. Furthermore, there was no interaction between frequency and time [Figure $2 \mathbf{A}$, right panel; $F(6,124)=2.07, p=0.06]$, indicating that the HT difference over time, possibly induced by anesthetics, was not frequencies dependent. In conclusion, the animals present a standard audiogram, and any change in the HT during the 2-h anesthesia may be due to the effects of the anesthetics themselves.

\section{The Hearing Threshold in the Salicylate Group}

The audiograms in the salicylate group (group $S, n=19$ ) were analyzed accordingly (Figure 2B) to find any effects of salicylate on the HT over time. Again, a one-factorial repeated 
measurement mixed ANOVA with the factor frequency and the repetition factor time and interaction of time $X$ frequency was calculated. In Figure $\mathbf{2 B}$, left panel, the mean audiogram (factor frequency) over time is depicted. Figure $\mathbf{2 B}$, center panel, shows the frequency-averaged HT over time in gerbils with salicylate injection. In this case, no HT difference over time was found (mean pre: $39.83 \pm 13.82 \mathrm{~dB}$ SPL; mean, 20 min post SS injection: $38.19 \pm 16.31 \mathrm{~dB}$ SPL; mean, $2 \mathrm{~h}$ post SS injection: $40.53 \pm 13.31 \mathrm{~dB} \mathrm{SPL}$ ), pointing to a possible effect of the salicylate that counteracts the thresholdreducing effect observed in the control group. In line with this interpretation, we observed a significant interaction of time $X$ frequency $[F(6,128)=5.15, p<0.001]$. With Tukey post hoc tests revealing no difference of $20 \mathrm{~min}$ post salicylate injection HT compared with pre-injection HT, but a significant HT increase at $4 \mathrm{kHz} 2 \mathrm{~h}$ post-injection compared with $20 \mathrm{~min}$ $(p=0.018)$ and pre-injection $(p<0.001)$. In other words, compared with the control group, no general HT improvement was found in the salicylate animals, but the contrary effect, i.e., a hearing loss, at the best hearing frequency was identified $2 \mathrm{~h}$ after the injection. Nevertheless, the described effect of the salicylate is transitory, as shown in Figure 2C. The onefactorial repeated measurement mixed ANOVA with the factor frequency and the repetition factor time and interaction of time $X$ frequency did not reveal any HT differences in the same $\mathrm{S}$ group animals 1 week post-injection compared with their pre-injection recordings of the HT.

\section{Comparison of HT Between the Salicylate and the Control Groups}

We compared the salicylate effect on the HT with the possible anesthetics effects in group $\mathrm{C}$ by two-factorial ANOVAs with the factors group and frequency and their interaction at the three different time points independently (Figure 3). While HT showed the typical frequency dependence [factor frequency; $F(3,137)=20.08, p<0.001]$, both groups did not show significantly different HT before the injection (C: $37.77 \pm 13.50 \mathrm{~dB}$ SPL; S: $39.98 \pm 13.72 \mathrm{~dB}$ SPL), and no significant interaction of time $X$ frequency emerged (Figure 3A). The same was true at $20 \mathrm{~min}$ after the injection [factor frequency; $F(3,133)=8.08, p<0.001]$, no difference between mean HT of both groups (C: C: $34.95 \pm 13.75 \mathrm{~dB}$ SPL; S: $38 \pm 16.12 \mathrm{~dB}$ SPL) and no significant interaction between the factors (Figure 3B). Nevertheless, after 2 h (Figure 3C, left panel) there was a significantly higher mean HT in the salicylate group compared with the control animals [C: $32.94 \pm 12.72 \mathrm{~dB}$ SPL; S: $39.51 \pm 13.53 \mathrm{~dB}$ SPL, $F(1,132)=9.92, p=0.002]$, and $\mathrm{a}$ significant interaction of both factors $[F(3,132)=2.72, p=0.047]$. At $4 \mathrm{kHz}$ (which represents the frequency range of best hearing in Mongolian gerbils), the HT was affected strongest, as indicated by a significant Tukey post hoc test $(p=0.003$, Figure 3C, right panel). These data clearly showed no difference in the HT of the animals before the injection of salicylate. Over time, the drug showed its effect with 20 min post-injection, the HT in both groups still being comparable, but, $2 \mathrm{~h}$ post injection, the HT of the salicylate group increased specifically at the best hearing frequency of the animals.
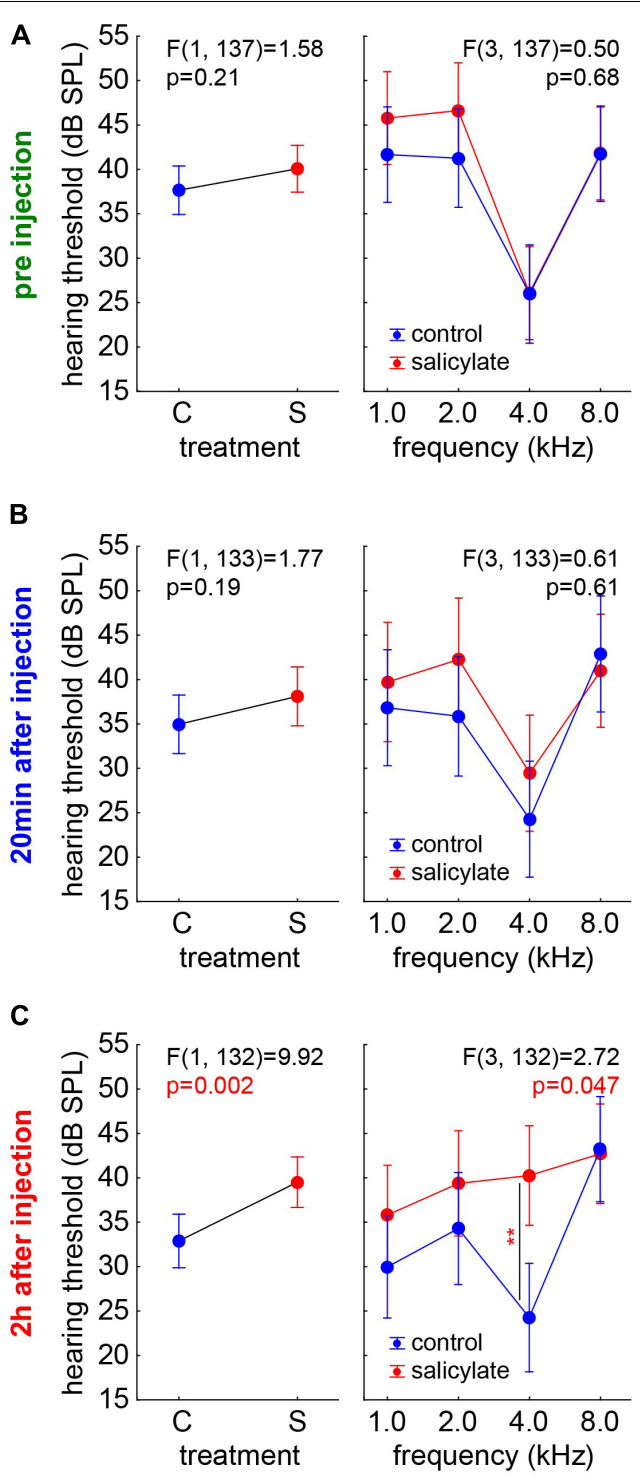

FIGURE 3 | Comparison of the HT of the salicylate and control groups over time. Results of the two-factorial ANOVAs with the factors group (left panels) and their interaction with frequency (right panels) at the three different time points. (A) Before the injection, both groups did not show significantly different $\mathrm{HT}$ and no significant interaction of time $X$ frequency. (B) 20 min after injection, there is no significant different $\mathrm{HT}$, and there is no significant interaction between the factors. (C) $2 \mathrm{~h}$ after injection, a significant higher $\mathrm{HT}$ in the salicylate group compared with the control animals and a significant interaction of both factors was found. At $4 \mathrm{kHz}$, the HT was affected strongest, as indicated by a significant Tukey post hoc test $(p=0.003)$.

\section{Hearing Loss in the Control Group}

To rule out any effect biases of single individuals, we reanalyzed the data, using not the HT but the HL ( $\left.\mathrm{HT}_{\text {post }}-\mathrm{HT}_{\text {pre }}\right)$, where positive values indicate worse $\mathrm{HT}_{\text {post }}$, negative values better $\mathrm{HT}_{\text {post }}$ compared with the pre measurements. As described above, we first analyzed control and salicylate animals with one factorial repeated measurement mixed ANOVAs with factor frequency, repetition factor time $(20 \mathrm{~min}$ and $2 \mathrm{~h}$ ) and the 
interaction of both factors (Figure 4). In the group C (Figure 4A), mostly negative HL values were found across all frequencies with a significant frequency dependency $(p=0.035)$ and a significant Tukey post hoc test when comparing $1 \mathrm{kHz}$ with $8 \mathrm{kHz}$ ( $p=0.048)$. No significant difference between the average HL of $20 \mathrm{~min}$ and $2 \mathrm{~h}$ was found (factor time: mean $20 \mathrm{~min}$ post saline injection: $-2.76 \pm 10.76 \mathrm{~dB}$; mean $2 \mathrm{~h}$ post saline injection: $-5.51 \pm 12.65 \mathrm{~dB}$ ). This was also true for the interaction of both factors $[F(3,62)=1.13, p=0.34]$, indicating better hearing at both time points, especially in lower frequency ranges. In other words, better hearing (negative HL) after control injections could be found specifically at lower frequencies, but no significant difference over the two time points emerged, indicating a then stable hearing level of the animals.

\section{Hearing Loss in the Salicylate Group}

In the group $S$ (Figure 4B), a significant frequency dependency of the HL was also found $(p=0.002)$ with a significant positive HL at $4 \mathrm{kHz}$ compared with the negative 1 and $2 \mathrm{kHz} \mathrm{HL}$ values (Tukey post hoc tests, $p=0.001$ and $p=0.041$ ). Again, no significant HL difference between the two time points was detected (mean, 20 min post salicylate injection: $-2.78 \pm 15.55 \mathrm{~dB}$; mean, $2 \mathrm{~h}$ post salicylate injection: $-0.44 \pm 15.68 \mathrm{~dB}$ ), but a significant interaction of time $X$ frequency $[F(3,64)=4.28, p<0.05]$ with a significant Tukey post hoc test at $4 \mathrm{kHz}(p=0.011)$ again indicated the strongest HL at the best hearing frequency after $2 \mathrm{~h}$ post salicylate injection.

\section{Comparison of HL Between the Control and the Salicylate Groups}

The comparison of the $\mathrm{HL}$ of groups $\mathrm{C}$ and $\mathrm{S}$ was again performed by two two-factorial ANOVAs with the factors group and frequency independent for both time points (Supplementary Figure 1). After $20 \mathrm{~min}$ (Supplementary Figure 1A) neither factor frequency $[F(3,131)=2.60, p=0.06]$ nor the factor group $[\mathrm{C}:-2.55 \pm 10.99 \mathrm{~dB}$; $\mathrm{S}:-2.92 \pm 15.49 \mathrm{~dB}, F(1,131)=0.06$, $p=0.80]$ showed any significant effect on the HL, which was also true for the interaction of both factors $(p=0.81)$. After $2 \mathrm{~h}$, on the other hand (Supplementary Figure 1B), significant frequency dependence could be identified $[F(3,132)=14.11, p<0.001]$ but no difference between both groups [C: $-5.51 \pm 12.65 \mathrm{~dB}$; $\mathrm{S}$ : $-1.35 \pm 15.98 \mathrm{~dB}, F(1,132)=3.42, p=0.07]$. Nevertheless, the significant interaction of both factors $[F(3,132)=3.20, p=0.025]$ and the significant Tukey post hoc test at $4 \mathrm{kHz}(p=0.007)$
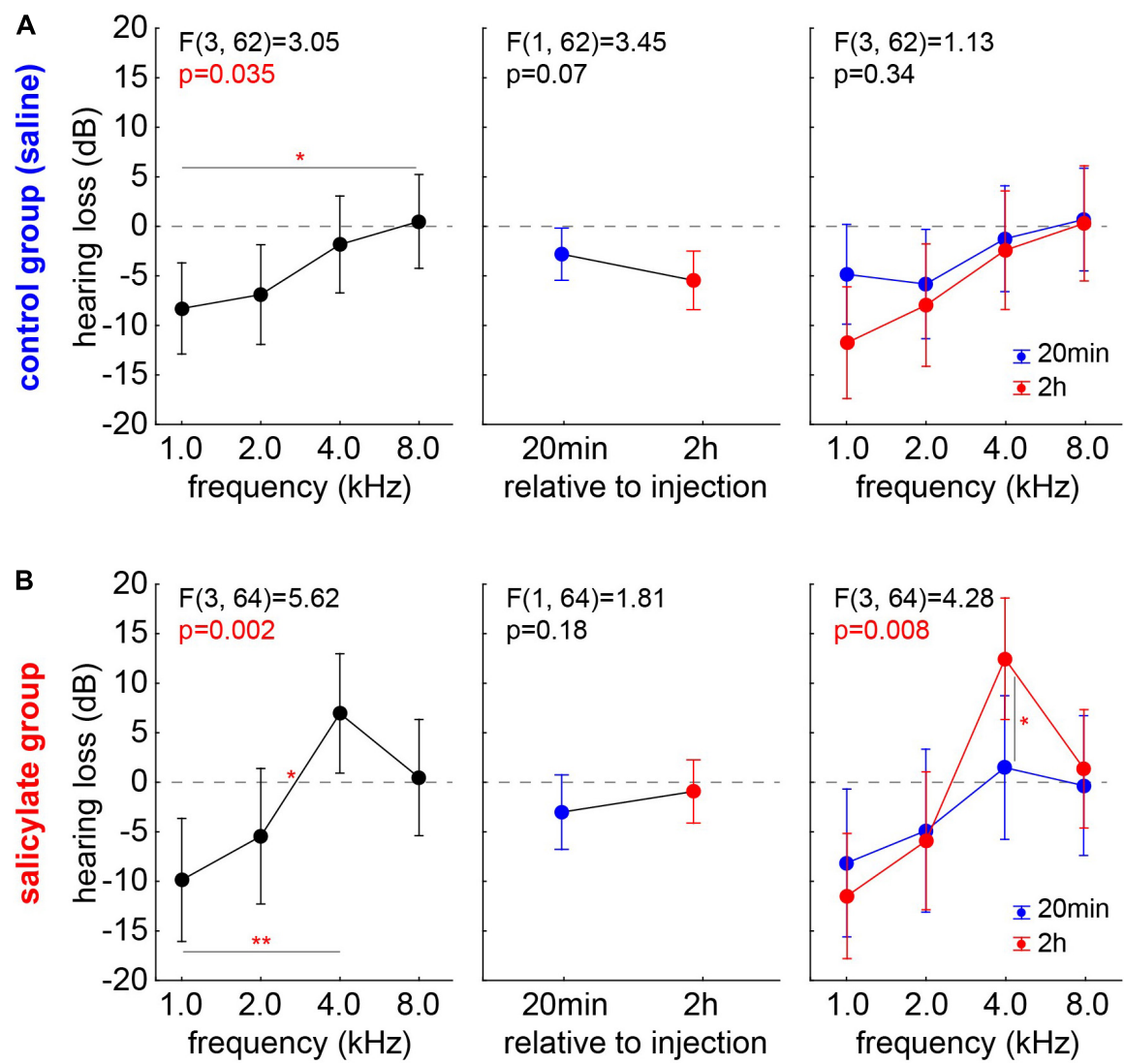

FIGURE 4 | HL in control and salicylate animals analyzed with one factorial repeated measurement mixed ANOVAs with factor frequency (left panels), repetition factor time (20 min and $2 \mathrm{~h}$; center panels) and the interaction of both factors (right panels). (A) In group C, mostly negative HL values could be found over all frequencies with significant frequency dependency but no significant difference between the average $\mathrm{HL}$ of $20 \mathrm{~min}$ and $2 \mathrm{~h}$ or in the interaction of the factors could be found. (B) In group S, significant frequency dependency of the HL was found, no significant HL difference between the two time points but a significant interaction of time $X$ frequency with a significant Tukey post hoc test at $4 \mathrm{kHz}$ emerged. ${ }^{*} p<0.05 ;{ }^{* *} p<0.01$. 
confirms the findings in the HT described above. So, neither 20 min nor $2 \mathrm{~h}$ after injection, a general significant effect of the injection on the HL could be identified. Nevertheless, $2 \mathrm{~h}$ after injection with salicylate, the HL data showed a specific increase at $4 \mathrm{kHz}$ only.

\section{Effects of Salicylate Injection on Behavioral Signs of Tinnitus Effect Size in the Control Group}

Animals of group $\mathrm{C}$ showed no behavioral signs of tinnitus in the GPIAS experiments after 20 min post saline injection $(n=9 ; 0 / 36$ $t$-tests with a significant negative effect size) and only in $4 / 36$ cases after $2 \mathrm{~h}$ after saline injection $(n=9)$. The $\mathrm{chi}^{2}$-test did not show a significant difference between these two time points. Independent of the significance of the effect size, it can be analyzed via a onefactorial repeated measurement mixed ANOVA with the factor frequency and the repetition factor time (Figure 5). No significant effect can be found in any of the factors, i.e., neither in the frequency $(p=0.96)$ nor in the factor time (mean 20 min post saline injection: $0.26 \pm 0.32$; mean, $2 \mathrm{~h}$ post saline injection: $0.31 \pm 0.45, F(1,68)=0.37, p=0.55)$ nor in the interaction $(p=0.13)$. In other words, we only see positive effect sizes that may indicate a cortical learning effect (cf. Discussion).

\section{Comparison of the Effect Size Between the Salicylate and the Control Groups}

The animals of the $S$ group already started to show first significantly negative effect sizes $(t$-tests, $p<0.05)$ after $20 \mathrm{~min}$ after salicylate injection $(n=10 ; 4 / 40)$ and doubled that value to eight cases $(n=19 ; 8 / 69)$ after $2 \mathrm{~h}$ after the injection. Still, the chi $^{2}$-test did not reveal a significant difference between both time points. We compared the effect sizes of both animal groups over the different frequencies by two two-factorial ANOVAs independently for the two time points (Figure 6). At $20 \mathrm{~min}$ after the injection (group C, $n=9$; group $S, n=10$ ) (Figure 6A), no significant effect of frequency on the effect size is found $[F(3,68)=2.11, p=0.11]$, which is also true for the effect size comparison across group (C:0.26 $\pm 0.32 ; \mathrm{S}: 0.11 \pm 0.43 ; p=0.10$ ) and the interaction of both factors $(p=0.53$ ). After $2 \mathrm{~h}$ (group
C, $n=9$; group $\mathrm{S}, n=19$ ), on the other hand (Figure $\mathbf{6 B}$ ), the effect size still did not depend on the frequency $[F(3,108)=0.27$, $p=0.85]$, but strongly depended on the group [C:0.31 \pm 0.45 ; S:0.003 $\pm 0.42, F(1,108)=14.40, p<0.001]$ and also showed a significant interaction $[F(3,108)=2.90, p=0.038$, with the Tukey post hoc test becoming significant at $4 \mathrm{kHz}(p=0.021)$. In other words, after $2 \mathrm{~h}$, we found a significantly lower effect size with negative values indicating a possible tinnitus percept - in salicylate animals compared with control animals. This difference is most prominent at $4 \mathrm{kHz}$, which is exactly the same frequency that shows strongest shifts toward higher HT in the ABR.

\section{Effects of Noise Trauma on ABR Thresholds and Behavioral Signs of Tinnitus}

For comparison, we analyzed ABR and GPIAS data from 16 animals before and after mild acoustic $2-\mathrm{kHz}$ trauma. Acute hearing loss (Figure 7A) and the effect size for tinnitus assessment (Figure 7B) were analyzed 1 week post trauma by two-factorial ANOVAs with the factors tinnitus animal group and stimulation frequency. We found not only a significant difference in HL and a strong trend in effect size between the animals with (T) and without tinnitus (NT) (Figures 7A,B, left panels) but also significant peaks at $4 \mathrm{kHz}$ in both measurements (center panels). As already published in our recent papers, $\mathrm{T}$ animals showed better hearing thresholds compared with NT animals. Especially in the effect size interaction of both factors, the significantly negative values at $4 \mathrm{kHz}$ (Tukey post hoc test, $p=0.003$ ) show that the behavioral changes are frequency dependent only in $\mathrm{T}$ animals, that is, in animals with negative effect size changes.

\section{Correlation of ABR and GPIAS Data in Salicylate and Trauma Animals}

To test if the underlying neurophysiological mechanisms of salicylate and trauma-induced tinnitus are similar, we investigated the correlations of the behavioral and (far-field) electrophysiological data by multiple linear regression analyses (Figure 8). In the trauma-induced tinnitus model, we have already demonstrated that stronger tinnitus percepts, as indicated by more negative effect sizes in the GPIAS, are correlated with lower HT, which is a prediction of the model of the SR mechanism
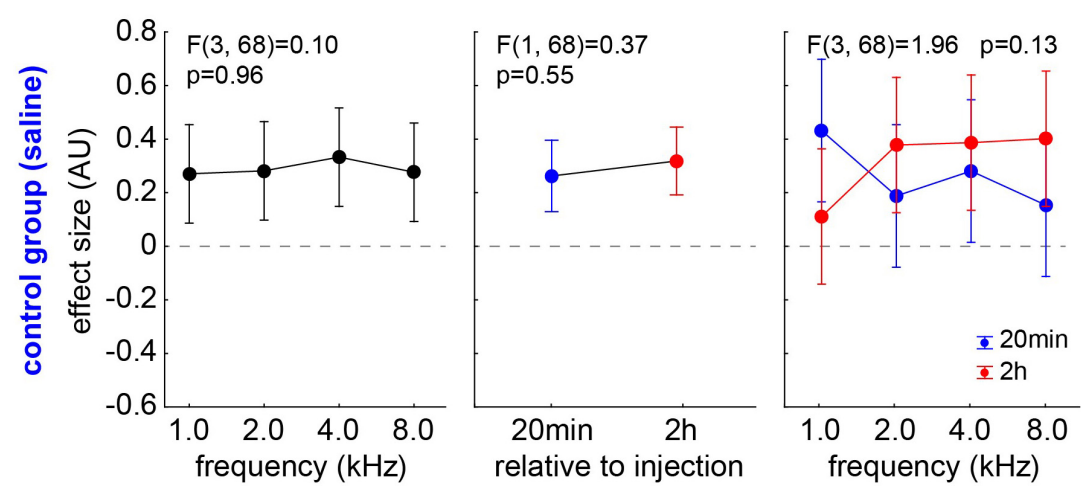

FIGURE 5 | Analysis of effect size $(\mathrm{AU})$ in group $\mathrm{C}$ by one-factorial repeated measurement mixed ANOVA with the factor frequency and the repetition factor time. No significant effect can be found in any of the factors. 

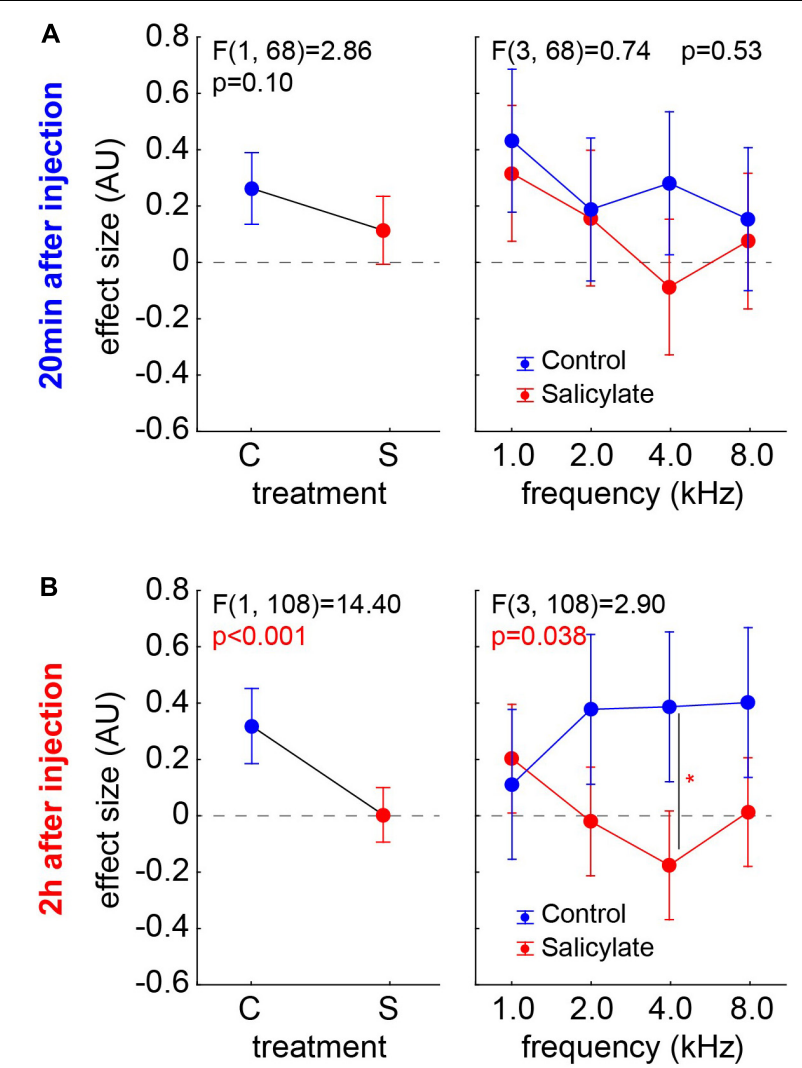

FIGURE 6 | Effect sizes of both animal groups (left panels) and their interaction with the factor frequencies (right panels) in two-factorial ANOVAs independent for the two time points. (A) 20 min after injection (group C, $n=9$; group $\mathrm{S}, n=10$ ) - no significant effects are found. (B) $2 \mathrm{~h}$ after injection (group C, $n=9$; group S, $n=19$ ), the effect size was strongly dependent on the group and also shows a significant interaction, with the Tukey post hoc test becoming significant at $4 \mathrm{kHz}(p=0.021)$.

for tinnitus development (cf. Krauss et al., 2016). In Figure 8A, the separate analyses for the animals in the $\mathrm{C}$ and $\mathrm{S}$ groups are depicted with their best linear fit for the correlation of effect size and HL after $2 \mathrm{~h}$ post-injection. Neither in the control group $\left(r^{2}=0.012, p=0.56\right)$ nor in the salicylate group $\left(r^{2}=0.001\right.$, $p=0.78$ ), a significant linear regression could be found. Figure $\mathbf{8 B}$ depicts the data of the 16 animals that received an acoustic trauma at $2 \mathrm{kHz}$ with $115 \mathrm{~dB}$ SPL over $75 \mathrm{~min}$. The correlation of the HL after 2-h post trauma and the subacute effect size measured after roughly 1 week showed a significant linear regression $\left(r^{2}=0.30, p<0.001\right)$, indicating that stronger tinnitus percepts (negative effect size values) lead to better HT (negative HL). In reverse conclusion, as the salicylate-induced tinnitus does not follow this pattern, it does not seem to rely on the same neurophysiological mechanism.

\section{DISCUSSION}

With this study, we aimed to investigate the neurophysiological mechanisms underlying salicylate and trauma-induced tinnitus.
To this end, the effect of salicylate on hearing thresholds measured by ABR and behavioral correlates of a tinnitus percept assessed by GPIAS was tested in Mongolian gerbils, and the results were compared to the same variables in animals with noise trauma-induced tinnitus. For the latter, we hypothesize the underlying neurophysiological mechanism to be based on auditory information optimization processes based on an SR mechanism (cf. Krauss et al., 2016). We found that salicylate induced behavioral changes associated with a possible tinnitus percept. However, due to the observed shifts in hearing threshold and correlation analyses of tinnitus strength and HT, this percept is most probably not produced by an SR-induced increase of neuronal activity but must be based on a different neuronal mechanism.

The model of SR that has been recently proposed in our group (Krauss et al., 2016, 2019) predicts that HT should be improved within the frequency range of the tinnitus percept. In accordance with the SR model, the audiometric data of almost 40,000 patients from the ENT clinic in Erlangen, tinnitus patients had significantly better HT than patients without tinnitus in the low-frequency range up to about $3 \mathrm{kHz}$, that is, in the speechrelevant frequency range (Krauss et al., 2016, 2019). Additionally, utilizing this mechanism, we proposed a therapeutic approach to tinnitus suppression, using external acoustic noise to replace the internal neuronal noise. In a pilot study, this approach was successful in patients with a hearing loss not exceeding $40 \mathrm{~dB}$ (Ahlf et al., 2012).

In the present study, we could demonstrate in an animal model that salicylate-induced transient tinnitus is most probably based on a different mechanism. Salicylate has been shown to act on the outer hair cell $(\mathrm{OHC})$ lateral wall stiffness (Lue and Brownell, 1999), increasing the membrane conductance of the OHCs (Stypulkowski, 1990) probably via acting on the voltage sensitivity of the motor protein prestin (Oliver et al., 2001; Grosh et al., 2004; Yu et al., 2008; Yang et al., 2009). Most likely, salicylate primarily influences electromotility and $\mathrm{OHC}$ nonlinear capacitance via a direct interaction with prestin (Greeson and Raphael, 2009). We speculate that this mechanism provides an explanation for the hearing loss induced by salicylate, but the mechanism and the site of the generation of the tinnitus percept still remain unclear (Guitton et al., 2003). The effects of salicylate are not only limited to the periphery, as a direct central effect of salicylate has also been demonstrated (Basta et al., 2008; Chen et al., 2013). Indeed, salicylate can easily get through the blood-brain barrier (Jastreboff et al., 1986) and change the delicate balance between the excitatory and inhibitory circuits in the central auditory system (Xu et al., 2005). In fact, synaptic inhibition of the auditory cortex is predominantly GABAergic (Prieto et al., 1994a,b) and an alteration of these circuits can greatly change the response properties of auditory neurons (Rajan, 1998; Wang et al., 2000, 2002) and could consequently cause tinnitus (Eggermont and Roberts, 2004; Eggermont, 2005). Instead, noise trauma-induced tinnitus could be caused by central changes arising from the noise-induced reduction of cochlear input (Norena et al., 2002; Eggermont, 2007; Moffat et al., 2009). In other words, noise trauma-induced tinnitus has to have a central origin as well. However, we hypothesize that it develops 

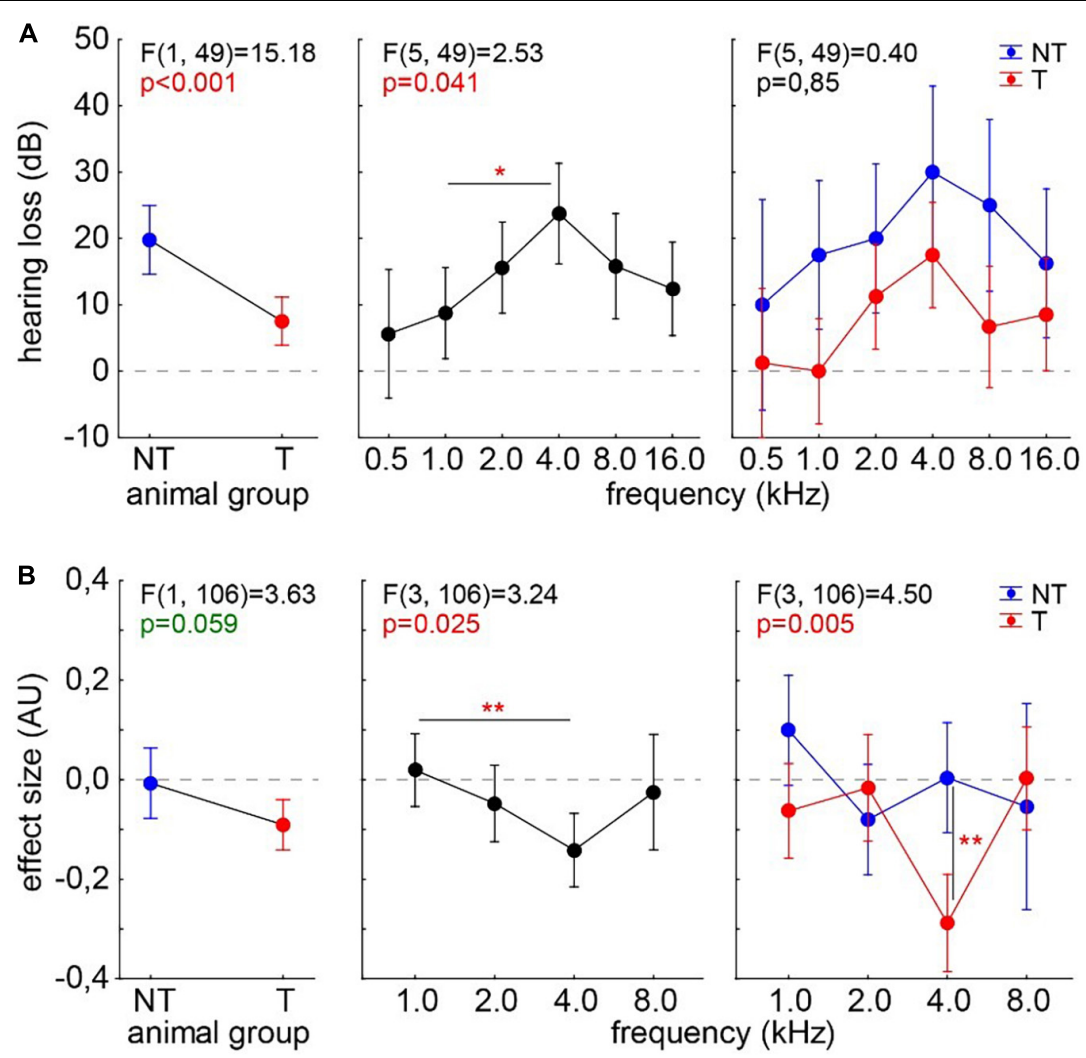

FIGURE 7 | Analyses of hearing loss (A) and GPIAS effect size (B) in 16 animals after an acoustic noise trauma centered around $2 \mathrm{kHz}$. The animals are separated into those with behavioral signs of tinnitus (T, $n=10$, red symbols) and those without such behavioral indications (NT, $n=6$, blue symbols). Given are the results of the two-factorial ANOVAs with factors animal group and stimulation frequency. Both analyses show a peak effect at $4 \mathrm{kHz}$. Asterisks indicate significant Tukey post hoc tests: ${ }^{\star} p<0.05,{ }^{\star *} p<0.01$.
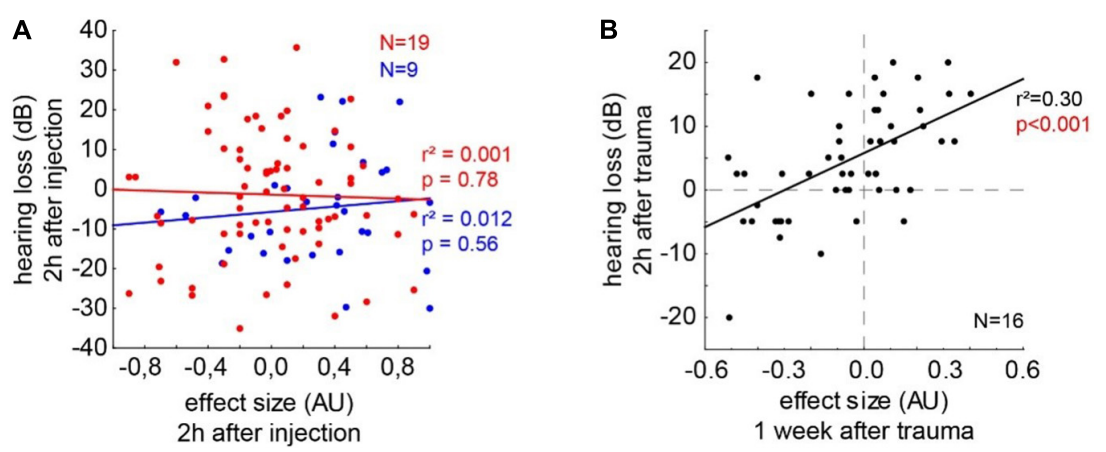

FIGURE 8 | Correlations of the behavioral and electrophysiological data by multiple linear regression analyses. (A) The separate analyses for the animals in the $\mathrm{C}$ and $\mathrm{S}$ groups are depicted with their best linear fit for the correlation of effect size and $\mathrm{HL}$ after $2 \mathrm{~h}$ post-injection. Neither in the control group $\left(r^{2}=0.012, p=0.56\right)$ nor in the salicylate group $\left(r^{2}=0.001, p=0.78\right)$ a significant linear regression could be found. (B) Depicts the data of the 16 animals that received an acoustic trauma at $2 \mathrm{kHz}$ with $115 \mathrm{~dB}$ SPL over $75 \mathrm{~min}$. The correlation of the $\mathrm{HL}$ after $2 \mathrm{~h}$ post trauma and the subacute effect size measured after roughly 1 week shows a significant linear regression $\left(r^{2}=0.30, p<0.001\right)$.

due to an indirect effect triggered by damage in the cochlea and not due to a direct effect in the brain. One could speculate that the direct central effect of the salicylate might also contribute to the difference in the frequency range between the broad tinnitus percept induced by salicylate and the narrow phantom percept induced by sound exposure (Norena et al., 2010; Ahlf et al., 2012).
For assessing any change in hearing sensitivity, we used $A B R$ measurements. In our data, the HT of the group C (Figure 2A) decreased over time in a frequency-independent manner. In that context, Ruebhausen and colleagues (Ruebhausen et al., 2012) noted that ABR generators were, primarily, in the central nervous system, and that interaction between general 
anesthesia and signal processing in the auditory brain stem would be expected. They conclude that, although both isoflurane and ketamine/xylazine were glutamatergic NMDA receptor antagonists, their global effect on neural systems would be complex and not known with sufficient precision to predict how each might affect auditory processing at a threshold. In our data, the HT before any kind of treatment (salicylate or saline) is comparable in groups $\mathrm{C}$ and $\mathrm{S}$ (Figure 3A). In group $\mathrm{C}$, we see a reduction of HT over time, while, in group $\mathrm{S}$, we do not observe this reduction, and we even find a frequencydependent increase of the $\mathrm{HT}$ at $4 \mathrm{kHz} 2 \mathrm{~h}$ post-injection (Figure 3B). This indicates that the effect of salicylate not only counteracts the reduction of HT but even increases the threshold in a frequency-specific manner. The reason why we see an effect only at $4 \mathrm{kHz}$ is probably due to the effect of the salicylate in the $\mathrm{OHC}$, which seems to be most prominent in the range of the best hearing. This means that the increase in the membrane conductance of the OHCs due to the effect of the salicylate would generate a stronger effect on the HT in the middle frequency region. Strikingly, this is not only a group effect (as demonstrated in the HT changed) but also an effect on an individual animal, as demonstrated by the HL at $4 \mathrm{kHz}$ (Figures 4A,B). Why this effect is so specific in the frequency range of best hearing, one can only speculate. It may be due to basilar membrane thickness that peaks around this frequency range (Plassmann et al., 1987), making it more stiff and, therefore, reducing the effectiveness of the weaker pull from the affected OHCs further, as force production, along the cochlea, seems to be similar (Mahendrasingam et al., 2010) and also the numbers and innervation of these cells (Wilson et al., 1991) do not seem to change over the course of cochlear frequency locations.

For the assessment of a possible tinnitus percept, we used the behavioral approach of the GPIAS paradigm. GPIAS is the most common method for tinnitus assessment because it does not require any training, avoids conditioning-related plasticity, and saves time (Turner et al., 2006). However, it is still controversial if the method is appropriate for tinnitus screening, as the "fillingin" interpretation has been questioned (Campolo et al., 2013; Radziwon et al., 2015). Furthermore, a wide range of criteria for positive tinnitus detection has been used across different laboratories, and there, still, is no consensus on a "best practice" for statistical evaluation of GPIAS results, as it exists for other behavioral paradigms (Hinkle et al., 2003). The method has also been strongly criticized for being not reliable and does not rule out the possibility detecting hearing loss rather than tinnitus. In order to overcome these limitations, Schilling and coworkers (Schilling et al., 2017) developed a new statistical approach based on the effect size of the behavioral response, used as a normalized measure for the PPI change. The method is robust and does not require any removal of outliers [which, otherwise, is a common practice (Longenecker and Galazyuk, 2011)]. The negative values of the effect size are easy to interpret and indicate less effect of the gap relative to the response of the startle pulse after treatment, which is considered to indicate a "filling" of the gap by a tinnitus percept in that frequency range and cannot be appointed to hearing loss alone. The method is, among others, applicable for salicylate or mono- or binaural noise trauma-induced tinnitus studies. We, here, see a clear effect of the salicylate $2 \mathrm{~h}$ but not $20 \mathrm{~min}$ post-injection. This is supported by the results of Jastreboff and coworkers (Jastreboff et al., 1986), who found that following i.p. injection of salicylate, the maximum levels in blood serum occurred after $1.5 \mathrm{~h}$, while the levels in the perilymph and spinal fluid reached their maximum within 2-4 h. Figures 5, 6 show the effect size, a normalized measure for the PPI change in the GPIAS (Hedges, 1982). If an animal has a stronger response to the gap (lower startle amplitude) during the post-recording compared to the precondition, the effect size will be positive. This may be due to proposed cortical learning effects that lead to increased responses to the startle (Moreno-Paublete et al., 2017). This phenomenon is always present, either when treating the animals with saline (Figure 5) or without treating the animals at all (unpublished data from our lab), or even when treating the animals with salicylate. On the other hand, the absolute values of the negative effect sizes can be interpreted as tinnitus severity, as it results from a smaller response to the gap, i.e., stronger startle amplitude due to potential "filling" of the gap by the tinnitus percept in the appropriate frequency range. The learning effects in the group $S$ can be seen in those frequency ranges where tinnitus is not so strongly perceived (Figure 6B, $1 \mathrm{kHz}$ ) while negative effect sizes dominate the $4 \mathrm{kHz}$ range. The consequence of the proposed cortical learning effect would be that the tinnitus percept must be strong enough to overcome the learning. In other words, we probably always underestimate the tinnitus percept, but, still, at $2 \mathrm{~h}$ post-injection, animals show a significant negative effect size compared to the control group at $4 \mathrm{kHz}$ (Figure 6B). This matches perfectly with our data of HT shift, indicating that the salicylate-induced HL and the tinnitus percept both lie in the best hearing frequency range.

In data of trauma animals (Figure 7), we found clear effects of HL dependency on the effect size of the behavioral measurements, i.e., on a significant tinnitus percept in a least one frequency or the lack thereof. Interestingly, while the maximum HL was centered around the frequency range of best hearing, comparable with the effect of salicylate, salicylate-induced tinnitus increases HL while trauma-induced tinnitus decreases the effect of the trauma on hearing thresholds.

Comparable between both tinnitus induction methods is the maximum effect size change at exactly the frequency of best hearing/maximal HL in tinnitus animals only. This indicates that the behavioral outcome of both methods is comparable.

To further demonstrate that the underlying neurophysiological mechanism - independent if our hypothesis is correct or not - is different for trauma and salicylate-induced tinnitus, we correlated the effect size with the HL for all the given frequencies after $2 \mathrm{~h}$ post the injection in the groups $\mathrm{C}$ and $\mathrm{S}$. As expected, in the group $\mathrm{C}$, no correlation between the two variables can be found (Figure $\mathbf{8 A}$, blue line) as, in this group, no tinnitus was induced. In the group $\mathrm{S}$, we also found no correlation between the tinnitus strength and the HL (Figure 8A, red line). In contrast, in noise trauma-induced tinnitus, a significant positive correlation could be found, indicating that, for strongest tinnitus percepts (negative effect size), hearing 
thresholds are improved (negative HL). We can, therefore, conclude that, in noise trauma/hearing loss-induced tinnitus in rodents, the tinnitus percept is most probably based on the neurophysiological mechanism of SR, but salicylate-induced tinnitus is not based on that same mechanism. The exact neurophysiological differences between both models of tinnitus induction have to be investigated in further studies.

\section{DATA AVAILABILITY STATEMENT}

The raw data supporting the conclusions of this article will be made available by the authors, without undue reservation.

\section{ETHICS STATEMENT}

The animal study was reviewed and approved by the Regierungspräsidium Unterfranken, Würzburg, Germany.

\section{REFERENCES}

Ahlf, S., Tziridis, K., Korn, S., Strohmeyer, I., and Schulze, H. (2012). Predisposition for and prevention of subjective tinnitus development. PLoS One 7:e44519. doi: 10.1371/journal.pone.0044519

Baguley, D., Axon, P., Winter, I., and Moffat, D. (2002). The effect of vestibular nerve section upon tinnitus. Clin. Otolaryngol. Allied Sci. 27, 219-226. doi: 10.1046/j.1365-2273.2002.00566.x

Barrs, D. M., and Brackmann, D. E. (1984). Translabyrinthine nerve section: effect on tinnitus. J. Laryngol. Otol. 98, 287-293. doi: 10.1017/s1755146300090648

Basta, D., Goetze, R., and Ernst, A. (2008). Effects of salicylate application on the spontaneous activity in brain slices of the mouse cochlear nucleus, medial geniculate body and primary auditory cortex. Hear. Res. 240, 42-51. doi: 10.1016/j.heares.2008.02.005

Campolo, J., Lobarinas, E., and Salvi, R. (2013). Does tinnitus "fill in" the silent gaps? Noise Health 15, 398-405. doi: 10.4103/1463-1741.121232

Cazals, Y. (2000). Auditory sensori-neural alterations induced by salicylate. Prog. Neurobiol. 62, 583-631. doi: 10.1016/s0301-0082(00)00027-7

Chao, Z., Qiuju, W., and Wei, S. (2014). Animal behavioral models of tinnitus. J. Otol. 9, 58-63. doi: 10.1016/s1672-2930(14)50016-5

Chen, G.-D., Stolzberg, D., Lobarinas, E., Sun, W., Ding, D., and Salvi, R. (2013). Salicylate-induced cochlear impairments, cortical hyperactivity and retuning, and tinnitus. Hear. Res. 295, 100-113. doi: 10.1016/j.heares.2012. 11.016

Chermak, G. D., and Dengerink, J. E. (1987). Characteristics of temporary noiseinduced tinnitus in male and female subjects. Scand. Audiol. 16, 67-73. doi: $10.3109 / 14992028709042158$

Coles, R. (1984). Epidemiology of tinnitus:(1) prevalence. J. Laryngol. Otol. 98, 7-15. doi: 10.1017/s1755146300090041

Day, R., Graham, G., Bieri, D., Brown, M., Cairns, D., Harris, G., et al. (1989). Concentration-response relationships for salicylate-induced ototoxicity in normal volunteers. Br. J. Clin. Pharmacol. 28, 695-702. doi: 10.1111/j.13652125.1989.tb03562.x

Eggermont, J. J. (2005). Tinnitus: neurobiological substrates. Drug Discov. Today 10, 1283-1290. doi: 10.1016/s1359-6446(05)03542-7

Eggermont, J. J. (2007). Pathophysiology of tinnitus. Prog. Brain Res. 166, 19-543. doi: 10.1016/s0079-6123(07)66002-6

Eggermont, J. J., and Roberts, L. E. (2004). The neuroscience of tinnitus. Trends Neurosci. 27, 676-682.

Gerken, G. M. (1996). Central tinnitus and lateral inhibition: an auditory brainstem model. Hear. Res. 97, 75-83. doi: 10.1016/s0378-5955(96)80009-8

Gerum, R., Rahlfs, H., Streb, M., Krauss, P., Grimm, J., Metzner, C., et al. (2019). Open (G) PIAS: an open-source solution for the construction of a highprecision acoustic startle response setup for tinnitus screening and threshold

\section{AUTHOR CONTRIBUTIONS}

VL collected the data. VL and KT analyzed the data. VL, KT, and HS wrote the manuscript. All authors contributed to the article and approved the submitted version.

\section{FUNDING}

This project has received funding from the European Research Council (ERC) under the European Union's Horizon 2020 Research and Innovation Program (grant agreement No. 764604).

\section{SUPPLEMENTARY MATERIAL}

The Supplementary Material for this article can be found online at: https://www.frontiersin.org/articles/10.3389/fnbeh. 2021.698516/full\#supplementary-material

estimation in rodents. Front. Behav. Neurosci. 13:140. doi: 10.3389/fnbeh.2019. 00140

Greeson, J. N., and Raphael, R. M. (2009). Amphipath-induced nanoscale changes in outer hair cell plasma membrane curvature. Biophys. J. 96, 510-520. doi: 10.1016/j.bpj.2008.09.016

Grosh, K., Zheng, J., Zou, Y., De Boer, E., and Nuttall, A. L. (2004). High-frequency electromotile responses in the cochlea. J. Acoust. Soc. Am. 115, 2178-2184. doi: $10.1121 / 1.1695431$

Guitton, M. J., Caston, J., Ruel, J., Johnson, R. M., Pujol, R., and Puel, J.-L. (2003). Salicylate induces tinnitus through activation of cochlear NMDA receptors. J. Neurosci. 23, 3944-3952. doi: 10.1523/jneurosci.23-09-03944.2003

Hedges, L. V. (1982). Estimation of effect size from a series of independent experiments. Psychol. Bull. 92:490. doi: 10.1037/0033-2909.92.2.490

Hinkle, D. E., Wiersma, W., and Jurs, S. G. (2003). Applied Statistics for the Behavioral Sciences. Boston, MA: Houghton Mifflin College Division.

House, J. W., and Brackman, D. (1981). Tinnitus: surgical treatment. Ciba Foundation Symposium 85, 204-212. doi: 10.1002/9780470720677.ch12

Jastreboff, P. J. (1990). Phantom auditory perception (tinnitus): mechanisms of generation and perception. Neurosci. Res. 8, 221-254. doi: 10.1016/01680102(90)90031-9

Jastreboff, P. J., Hansen, R., Sasaki, P. G., and Sasaki, C. T. (1986). Differential uptake of salicylate in serum, cerebrospinal fluid, and perilymph. Arch. Otolaryngol-Head Neck Surg. 112, 1050-1053. doi: 10.1001/archotol.1986. 03780100038004

Krauss, P., Schilling, A., Tziridis, K., and Schulze, H. (2019). Models of tinnitus development: from cochlea to cortex. HNO 67, 172-177.

Krauss, P., Tziridis, K., Metzner, C., Schilling, A., Hoppe, U., and Schulze, H. (2016). Stochastic resonance controlled upregulation of internal noise after hearing loss as a putative cause of tinnitus-related neuronal hyperactivity. Front. Neurosci. 10:597. doi: 10.3389/fnins.2016.00597

Langguth, B., Landgrebe, M., Kleinjung, T., Sand, G. P., and Hajak, G. (2011) Tinnitus and depression. World J. Biol. Psychiatry 12, 489-500.

Leaver, A. M., Turesky, T. K., Seydell-Greenwald, A., Morgan, S., Kim, H. J., and Rauschecker, J. P. (2016). Intrinsic network activity in tinnitus investigated using functional MRI. Hum. Brain Mapp. 37, 2717-2735. doi: 10.1002/hbm. 23204

Lewis, J. E., Stephens, S., and McKenna, L. (1994). Tinnitus and sucide. Clin Otolaryngol. Allied Sci. 19, 50-54. doi: 10.1111/j.1365-2273.1994.tb01147.x

Longenecker, R. J., and Galazyuk, A. V. (2011). Development of tinnitus in CBA/CaJ mice following sound exposure. J. Assoc. Res. Otolaryngol. 12:647. doi: 10.1007/s10162-011-0276-1

Lue, A. J.-C., and Brownell, W. E. (1999). Salicylate induced changes in outer hair cell lateral wall stiffness. Hear. Res. 135, 163-168. doi: 10.1016/s0378-5955(99) 00102-1 
Mahendrasingam, S., Beurg, M., Fettiplace, R., and Hackney, C. M. (2010). The ultrastructural distribution of prestin in outer hair cells: a post-embedding immunogold investigation of low-frequency and high-frequency regions of the rat cochlea. Eur. J. Neurosci. 31, 1595-1605. doi: 10.1111/j.1460-9568.2010. 07182.x

McFadden, D., Plattsmier, H., and Pasanen, E. (1984). Aspirin-induced hearing loss as a model of sensorineural hearing loss. Hear. Res. 16, 251-260. doi: 10.1016/0378-5955(84)90114-x

Metternich, F., and Brusis, T. (1999). Acute hearing loss and tinnitus caused by amplified recreational music. Laryngorhinootologie 78, 614-619.

Moffat, G., Adjout, K., Gallego, S., Thai-Van, H., Collet, L., and Norena, A. (2009). Effects of hearing aid fitting on the perceptual characteristics of tinnitus. Hear. Res. 254, 82-91. doi: 10.1016/j.heares.2009.04.016

Moreno-Paublete, R., Canlon, B., and Cederroth, C. R. (2017). Differential neural responses underlying the inhibition of the startle response by pre-pulses or gaps in mice. Front. Cell. Neurosci. 11:19. doi: 10.3389/fncel.2017.00019

Mrena, R., Savolainen, S., Kuokkanen, J. T., and Ylikoski, J. (2002). Characteristics of tinnitus induced by acute acoustic trauma: a long-term follow-up. Audiol. Neurotol. 7, 122-130. doi: 10.1159/000057660

Myers, E. N., and Bernstein, J. M. (1965). Salicylate ototoxicity: a clinical and experimental study. Arch. Otolaryngol. 82, 483-493. doi: 10.1001/archotol. 1965.00760010485006

Norena, A., Micheyl, C., Chéry-Croze, S., and Collet, L. (2002). Psychoacoustic characterization of the tinnitus spectrum: implications for the underlying mechanisms of tinnitus. Audiol. Neurotol. 7, 358-369. doi: 10.1159/000066156

Norena, A., Moffat, G., Blanc, J., Pezard, L., and Cazals, Y. (2010). Neural changes in the auditory cortex of awake guinea pigs after two tinnitus inducers: salicylate and acoustic trauma. Neuroscience 166, 1194-1209. doi: 10.1016/j. neuroscience.2009.12.063

Noreña, A. J., and Farley, B. J. (2013). Tinnitus-related neural activity: theories of generation, propagation, and centralization. Hear. Res. 295, 161-171. doi: 10.1016/j.heares.2012.09.010

Oliver, D., He, D. Z., Klöcker, N., Ludwig, J., Schulte, U., Waldegger, S., et al. (2001). Intracellular anions as the voltage sensor of prestin, the outer hair cell motor protein. Science 292, 2340-2343. doi: 10.1126/science.1060939

Plassmann, W., Peetz, W., and Schmidt, M. (1987). The cochlea in gerbilline rodents. Brain Behav. Evol. 30, 82-102. doi: 10.1159/0001 18639

Prieto, J. J., Peterson, B. A., and Winer, J. A. (1994a). Laminar distribution and neuronal targets of GABAergic axon terminals in cat primary auditory cortex (AI). J. Comp. Neurol. 344, 383-402. doi: 10.1002/cne.9034 40305

Prieto, J. J., Peterson, B. A., and Winer, J. A. (1994b). Morphology and spatial distribution of GABAergic neurons in cat primary auditory cortex (AI). J. Comp. Neurol. 344, 349-382. doi: 10.1002/cne.903440304

Pulec, J. L. (1995). Cochlear nerve section for intractable tinnitus. Ear Nose Throat J. 74, 468-476. doi: 10.1177/014556139507400708

Radziwon, K. E., Stolzberg, D. J., Urban, M. E., Bowler, R. A., and Salvi, R. J. (2015). Salicylate-induced hearing loss and gap detection deficits in rats. Front. Neurol. 6:31. doi: 10.3389/fneur.2015.00031

Rajan, R. (1998). Receptor organ damage causes loss of cortical surround inhibition without topographic map plasticity. Nat. Neurosci. 1, 138-143. doi: 10.1038/388

Ruebhausen, M., Brozoski, T., and Bauer, C. (2012). A comparison of the effects of isoflurane and ketamine anesthesia on auditory brainstem response (ABR) thresholds in rats. Hear. Res. 287, 25-29. doi: 10.1016/j.heares.2012.04.005

Ryan, A. (1976). Hearing sensitivity of the mongolian gerbil. M erionesunguiculatis. J. Acoust. Soc. Am. 59, 1222-1226. doi: 10.1121/1.380961

Schaette, R., and McAlpine, D. (2011). Tinnitus with a normal audiogram: physiological evidence for hidden hearing loss and computational model. J. Neurosci. 31, 13452-13457. doi: 10.1523/jneurosci.2156-11.2011

Schilling, A., Gerum, R., Krauss, P., Metzner, C., Tziridis, K., and Schulze, H. (2019). Objective estimation of sensory thresholds based on neurophysiological parameters. Front. Neurosci. 13:481. doi: 10.3389/fnins.2019.00481

Schilling, A., Krauss, P., Gerum, R., Metzner, C., Tziridis, K., and Schulze, H. (2017). A new statistical approach for the evaluation of gap-prepulse inhibition of the acoustic startle reflex (GPIAS) for tinnitus assessment. Front. Behav. Neurosci. 11:198. doi: 10.3389/fnbeh.2017.00198

Schilling, A., Krauss, P., Hannemann, R., Schulze, H., and Tziridis, K. (2020). Reduktion der Tinnituslautstärke. HNO. doi: 10.1007/s00106-020-00963-5
Shargorodsky, J., Curhan, G. C., and Farwell, W. R. (2010). Prevalence and characteristics of tinnitus among US adults. Am. J. Med. 123, 711-718. doi: 10.1016/j.amjmed.2010.02.015

Sheppard, A., Hayes, S., Chen, G.-D., Ralli, M., and Salvi, R. (2014). Review of salicylate-induced hearing loss, neurotoxicity, tinnitus and neuropathophysiology. Acta Otorhinolaryngol. Ital. 34:79.

Silverstein, H., Haberkamp, T., and Smouha, E. (1986). The state of tinnitus after inner ear surgery. Otolaryngology-Head Neck Surg. 95, 438-441. doi: 10.1177/ 019459988609500404

Stankiewicz, C., Przewoźny, T., and Kozłowski, J. (2000). Noise from a car airbag as a cause of acute acoustic trauma. Otolaryngol. Polska 54, 775-781.

Stypulkowski, P. H. (1990). Mechanisms of salicylate ototoxicity. Hear. Res. 46, 113-145. doi: 10.1016/0378-5955(90)90144-e

Temmel, A., Kierner, A., Steurer, M., Riedl, S., and Innitzer, J. (1999). Hearing loss and tinnitus in acute acoustic trauma. Wiener Klinische Wochenschrift 111, 891-893.

Turner, J. G., Brozoski, T. J., Bauer, C. A., Parrish, J. L., Myers, K., Hughes, L. F., et al. (2006). Gap detection deficits in rats with tinnitus: a potential novel screening tool. Behav. Neurosci. 120:188. doi: 10.1037/0735-7044.120.1.188

Tziridis, K., Ahlf, S., Jeschke, M., Happel, M. F., Ohl, F. W., and Schulze, H. (2015). Noise trauma induced neural plasticity throughout the auditory system of Mongolian gerbils: differences between tinnitus developing and non-developing animals. Front. Neurol. 6:22. doi: 10.3389/fneur.2015.00022

Tziridis, K., Korn, S., Ahlf, S., and Schulze, H. (2014). Protective effects of Ginkgo biloba extract EGb 761 against noise trauma-induced hearing loss and tinnitus development. Neural Plast. 2014:427298.

Vanneste, S., and De Ridder, D. (2016). Deafferentation-based pathophysiological differences in phantom sound: tinnitus with and without hearing loss. Neuroimage 129, 80-94. doi: 10.1016/j.neuroimage.2015.12.002

Walter, M., Tziridis, K., Ahlf, S., and Schulze, H. (2012). Context dependent auditory threshold determined by brainstem audiometry and prepulse inhibition in mongolian gerbils. Open J. Acoustics 2, 34-49. doi: 10.4236/oja. 2012.21004

Wang, J., Caspary, D., and Salvi, R. J. (2000). GABA-A antagonist causes dramatic expansion of tuning in primary auditory cortex. Neuroreport 11, 1137-1140. doi: 10.1097/00001756-200004070-00045

Wang, J., McFadden, S. L., Caspary, D., and Salvi, R. (2002). Gamma-aminobutyric acid circuits shape response properties of auditory cortex neurons. Brain Res. 944, 219-231. doi: 10.1016/s0006-8993(02)02926-8

Wilson, J., Henson, M., and Henson, O. Jr. (1991). Course and distribution of efferent fibers in the cochlea of the mouse. Hear. Res. 55, 98-108. doi: 10.1016/ 0378-5955(91)90096- $\mathrm{r}$

Xu, H., Gong, N., Chen, L., and Xu, T.-L. (2005). Sodium salicylate reduces gamma aminobutyric acid-induced current in rat spinal dorsal horn neurons. Neuroreport 16, 813-816. doi: 10.1097/00001756-200505310-00007

Yang, K., Huang, Z.-W., Liu, Z.-Q., Xiao, B.-K., and Peng, J.-H. (2009). Long-term administration of salicylate enhances prestin expression in rat cochlea. Int. J. Audiol. 48, 18-23. doi: 10.1080/14992020802327998

Yu, N., Zhu, M.-L., Johnson, B., Liu, Y.-P., Jones, R., and Zhao, H.-B. (2008). Prestin up-regulation in chronic salicylate (aspirin) administration: an implication of functional dependence of prestin expression. Cell. Mol. Life Sci. 65, 2407-2418. doi: 10.1007/s00018-008-8195-y

Conflict of Interest: The authors declare that the research was conducted in the absence of any commercial or financial relationships that could be construed as a potential conflict of interest.

Publisher's Note: All claims expressed in this article are solely those of the authors and do not necessarily represent those of their affiliated organizations, or those of the publisher, the editors and the reviewers. Any product that may be evaluated in this article, or claim that may be made by its manufacturer, is not guaranteed or endorsed by the publisher.

Copyright (C) 2021 Lanaia, Tziridis and Schulze. This is an open-access article distributed under the terms of the Creative Commons Attribution License (CC BY). The use, distribution or reproduction in other forums is permitted, provided the original author(s) and the copyright owner(s) are credited and that the original publication in this journal is cited, in accordance with accepted academic practice. No use, distribution or reproduction is permitted which does not comply with these terms. 